\title{
Dank an die Gutachter
}

Sportwiss $2013 \cdot 43: 82$

DOI 10.1007/s12662-013-0280-1

Online publiziert: 22. Februar 2013

(c) Springer-Verlag Berlin Heidelberg 2013

Für die Qualität und Objektivität der Beiträge sind neben den engagierten Autorinnen und Autoren auch die vielen qualifizierten Gutachter maßgeblich, die im Rahmen des Peer-Review-Prozesses die Manuskripte inhaltlich-wissenschaftlich prüfen und Empfehlungen zur konkreten Verbesserung äußern. Allen Gutachtern, die im Jahr $2012 \mathrm{Ma}-$ nuskripte für die Sportwissenschaft begutachtet haben, danken wir herzlich für Ihre konstruktive und gewissenhafte Arbeit:

- Silvia Achtzehn

- Günter Amesberger

- Georg Anders

- Eckart Balz

- Christoph Bertling

- Alex Bertrams

- Thomas Bezold

- Tim Bindel

- Michael Brach

- Michael Bräutigam

- Martin-Peter Büch

- Ulrike Burrmann

- Dirk Büsch

- Achim Conzelmann

- Frank Daumann

- Helmut Digel

- Rosa Diketmueller

- Gunnar Drexel

- Sabine Eichberg

- Julia Everke

- Oliver Faude

- Ingo Froböse

- Armin Fuchs

- Holger Gabriel

- Petra Gieß-Stüber

- Andre Gogoll

- Wiebke Göhner

- Bernd Gröben

- Arne Güllich

- Norbert Hagemann

- Frank Hänsel
- Ulrich Hartmann

- Sandra Heck

- Christian Herrmann

- Antje Hoffmann

- Kirsten Hötting

- Petra Jansen

- Daniela Kahlert

- Markus Klein

- Verena Klusmann

- Stefan König

- Arnd Krüger

- Detlef Kuhlmann

- Stefan Künzell

- Martin Lames

- Markus Lamprecht

- Heinz Mechling

- Henk Erik Meier

- Roland Messmer

- Hermann Mueller

- Carl Müller-Platz

- Siegfried Nagel

- Nils Neuber

- Jeannine Ohlert

- Norbert Olivier

- Claudia Pawlenka

- Tim Pawlowski

- Gertrud Pfister

- Michael Pfitzner

- Werner Pitsch

- Markus Raab

- Kai Reinhart
- Marcel Reinold

- Kai Röcker

- Alfred Rütten

- Sabine Schäfer

- Friederike ScharhagRosenberger

- Nele Schlapkohl

- Wolfgang Schlicht

- Mirko Schmidt

- Jörg Schorer

- Nadja Schott

- Manfred Schubert

- Geoffrey Schweizer

- Theo Stemper

- Oliver Stoll

- Jana Strahler

- Gorden Sudeck

- Ralf Sygusch

- Jörg Thiele

- Gerd Thienes

- Markus Tilp

- Klaus Völker

- Vera Volkmann

- Matthias Wagner

- Petra Wagner

- Manfred Wegner

- Jon P.Wehrlin

- Josef Wiemeyer

- Alexander Woll

- Karen Zentgraf 